\title{
Vierfachtablette gegen Bluthochdruck
}

\section{Vier Wirkstoffe in einer Tablette: Das ist vermutlich gut für die Compliance der Patienten. Wie gut die Blutdrucksenkung mit einer Vierfachtablette funktioniert, wurde in einer kleinen Studie untersucht.}

$\mathrm{D}$ ie vorliegende Untersuchung ist eine randomisierte, placebokontrollierte doppelblinde Cross-over-Studie mit einer Vierfachtablette gegen Bluthochdruck. Die Tablette enthielt eine niedrige Dosis der Wirkstoffe Irbesartan (37,5 mg), Amlodipin (1,25 mg), Hydrochlorothiazid $(6,25 \mathrm{mg})$ und Atenolol $(12,5 \mathrm{mg})$. Die Teilnehmer der Studie hatten eine unbehandelte Hypertonie und wurden aus vier australischen Allgemeinpraxen rekrutiert. Sie erhielten randomisiert entweder die Vierfachtablette oder Placebo für vier Wochen. Nach einer anschließenden zweiwöchigen Auswaschphase erhielten die Patienten jeweils die andere Behandlungsform. Der primäre Endpunkt war der ambulant gemessene 24-Stunden-Blutdruck nach vier Wochen, korrigiert um den Placeboeffekt.

18 Patienten konnten ausgewertet werden. Die Vierfachtablette reduzierte den systolischen 24-Stunden-Blutdruck nach Korrektur um den Placeboeffekt um $19 \mathrm{mmHg}$ ( $95 \%$-Konfidenzintervall 14-23 mmHg). Der Sprechstundenblutdruck wurde um 22/13 $\mathrm{mmHg}$ gesenkt $(\mathrm{p}<0,0001)$. Während der Behandlung erreichten alle Teilnehmer eine Senkung des Sprechstundenblutdrucks unter 140/90 mmHg, während nur $33 \%$ der Patienten aus der Placebogruppe eine solche Blutdrucksenkung zeigten $(\mathrm{p}=$ 0,0013). Es gab keine schweren Nebenwirkungen der Behandlung.

Chow CK et al. Quarter-dose quadruple combination therapy for initial treatment of hypertension: placebo-controlled, crossover, randomised trial and systematic review. Lancet. 2017;389 (10073):1035-1042.

\section{Kommentar}

Diese Studie ist ein interessanter Versuch, den bisher in der Hypertoniebehandlung beschrittenen Weg der Kombinationsthera- pie weiter auszubauen. In der Vierfachtablette sind die jeweiligen Wirkstoffe in einer Dosierung enthalten, die bei einer Monotherapie sehr wahrscheinlich nur einen geringen Effekt hätte. Offenbar bewirkt die Vierfachkombination dennoch eine ausreichende Blutdrucksenkung.

Es liegt auf der Hand, dass diese niedrige Dosierung der einzelnen Substanzen das Risiko von Nebenwirkungen gegenüber den jetzt verfügbaren Kombinationen weiter vermindern dürfte.

Die Probandenzahl dieser Studie ist gering, sodass sie allenfalls als Pilotstudie anzusehen ist, der weitere, größere Studien folgen sollten. Falls sich die Befunde bestätigen, mag dies ein Weg zur nebenwirkungsfreien Hypertoniebehandlung sein. Nach wie vor sind Nebenwirkungen ein wichtiges Hindernis der Compliance in der Hypertoniebehandlung.

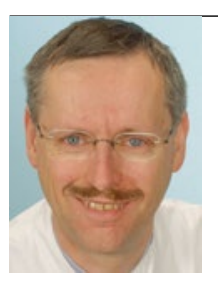

Prof. Dr. med. Walter Zidek Medizinische Klinik IV, Charité, Universitätsmedizin Berlin

\section{Gesundheitlicher Vorteil von Vollkornprodukten: Mythos oder evidenzbasiert?}

\section{Vollkornprodukte werden oftmals als "gesund" gepriesen, obwohl inzwischen nicht wenige Studien mit kontroversen Ergebnissen vorliegen. Nun erschien eine neue Metaanalyse mit umfangreichem Datenmaterial und differenzierter Auswertung.}

nsgesamt 45 Studien (Kohorten) aus Europa, den USA und Asien fanden Eingang in die Metaanalyse. Die Anzahl der Probanden in den einzelnen Studien lag je nach untersuchter Krankheit im Bereich von 245.012 bis 705.252.

Die folgenden Angaben beziehen sich auf einen Konsum von $90 \mathrm{~g}$ Vollkornprodukten pro Tag im Vergleich zu einem niedrigeren Konsum: Reduktion der Neuerkrankungsrate einer koronaren Herzkrankheit um $21 \%$, eines Schlaganfalls um $13 \%$, von kardiovaskulären Krankheiten um 16\% und von Krebser- krankungen um $15 \%$. Bei höherem Konsum an Vollkornprodukten zeigten sich höhere Reduktionen der Häufigkeit von koronarer Herzkrankheit und anderen kardiovaskulären Krankheiten, nicht jedoch beim Schlaganfall und beim Diabetes.

Auch eine Reduktion der Sterblichkeit war feststellbar. Die Gesamtsterblichkeit war um $17 \%$ niedriger, die Sterblichkeit aufgrund von respiratorischen Krankheiten um $22 \%$, aufgrund von Infektionskrankheiten um $26 \%$ und aufgrund von Diabetes um $51 \%$. Ein Kon- sum von $200 \mathrm{~g}$ Vollkornprodukten pro Tag reduzierte die Gesamtsterblichkeit um $28 \%$.

Aune $D$ et al. Whole grain consumption and risk of cardiovascular disease, cancer, and all cause mortality and cause specific mortality: systematic review and dose-response meta-analysis of prospective studies. BMJ. 2016;353:2716-2730.

\section{Kommentar}

Vollkorn besteht aus Getreide, das von Grannen und Spelzen befreit wurde. Neben dem Mehlkörper (Endosperm) und dem Keimling besteht es aus der Fruchtschale (Kleie). Im Unterschied zum weißen Mehl enthält Vollkornmehl die Fruchtschale, die aus Ballaststoffen, Vitaminen, Mineralien und Ölen besteht.

Die positiven gesundheitlichen Aspekte von Vollkornprodukten werden vor allem den Ballaststoffen zugeschrieben, Stoffe (z. B. Zellulosen, Pektine, Lignine), die von menschlichen Verdauungsenzymen nicht 